\title{
Tuberculosis reactivation at ileum following immune checkpoint inhibition with pembrolizumab for metastatic nasopharyngeal carcinoma: a case report
}

\author{
Kin-Sang Lau ${ }^{1 \dagger}$, Ben Man-Fei Cheung ${ }^{1 \dagger}$, Ka-On Lam¹, Sum-Yin Chan ${ }^{1}$, Ka-Ming Lam¹ , Chun-Fai Yeung ${ }^{2}$, \\ Ivan Fan-Ngai Hung ${ }^{3}$, Dora Lai-Wan Kwong ${ }^{1}$, Chi-Chung Tong ${ }^{1}$, To-Wai Leung ${ }^{1}$, Mai-Yee Luk', \\ Anne Wing-Mui Lee ${ }^{1}$, Kwok-Keung Yuen ${ }^{1}$ and Victor Ho-Fun Lee ${ }^{1 *}$ (D)
}

\begin{abstract}
Background: Tuberculosis (TB) reactivation has been increasingly identified following immune checkpoint inhibitor (ICI) therapy for cancer patients. However there has been no report on TB reactivation in the gastrointestinal tract. In the report, we describe a patient who developed TB ileitis after pembrolizumab for her metastatic nasopharyngeal carcinoma (NPC). Rechallenge with pembrolizumab after its temporary interruption together with anti-TB therapy produced continuous tumor response but without further TB reactivation.

Case presentation: A 29-year-old lady with metastatic NPC involving the cervical nodes, lungs and bones started pembrolizumab after failure to multiple lines of chemotherapy. She complained of sudden onset of abdominal pain, vomiting and bloody diarrhea with mucus 21 months after pembrolizumab. Colonoscopy revealed terminal ileitis with multiple caseating granulomas with Langerhan cells. Serum interferon gamma release assay was strongly positive. She was treated with anti-TB medication and was later rechallenged with pembrolizumab for her progressive lung metastases without further TB relapse while her lung metastases were brought under control again.

Conclusion: To date, this is the first gastrointestinal TB reactivation after ICl therapy for cancer. Guidelines to screen for TB before initiation of ICls in endemic areas should be established.
\end{abstract}

Keywords: Nasopharyngeal carcinoma, Immune checkpoint inhibitors, Tuberculosis reactivation, IGRA

\section{Background}

Immune checkpoint inhibitors (ICI) against programmed death-(ligand)1 (PD-(L)1) and cytotoxic T-lymphocyteassociated antigen 4 have gained increasing popularity for various malignancies [1]. Despite improvement in

\footnotetext{
*Correspondence: vhflee@hku.hk

${ }^{\dagger}$ Kin-Sang Lau and Ben Man-Fei Cheung contributed equally to this manuscript

${ }^{1}$ Department of Clinical Oncology, LKS Faculty of Medicine, The University of Hong Kong, Hong Kong, China

Full list of author information is available at the end of the article
}

progressive-free survival and overall survival particularly in melanoma and lung cancer, immune-related adverse events (irAE) have also been increasingly reported [2]. There has a dozen of case reports of tuberculosis (TB) reactivation after ICI therapy so far, including two patients who developed pulmonary $\mathrm{TB}$ reactivation during ICI therapy for their metastatic nasopharyngeal carcinoma (NPC) [3-15]. However, the incidence of $\mathrm{TB}$ reactivation after ICI therapy is western countries is exceedingly rare [16]. Here, we report a patient with metastatic NPC who developed TB ileitis during 
pembrolizumab therapy and was subsequently treated with a full course of anti-TB antibiotics with temporary ICI interruption. She was successfully re-challenged with pembrolizumab during anti-TB treatment, with her tumours still in continuous response. Various strategies on identifying latent TB infection before initiation of anti-TB prophylaxis are proposed.

\section{Case presentation}

A 29-year-old lady was diagnosed with metastatic NPC with cervical nodal, lung and bone metastases in December 2014. Her disease progressed despite multiple lines of chemotherapy including gemcitabine and cisplatin (3 cycles from December 2014 to March 2015)cisplatin plus 5-fluorouracil (1 cycle in April 2015) which was changed to docetaxel plus cisplatin because of 5-fluorouracil allergy (3 cycles from April 2015 to June 2015), gemcitabine and carboplatin (6 cycles from November 2015 to March 2016) capecitabine (6 cycles from April 2016 to August 2016), and metronomic cyclophosphamide (from August 2016 to September 2016). Radical chemoradiation with cisplatin was given to her progressive neck nodes in July 2015. Her lung metastases later further progressed resulting in mild dyspnea and her plasma Epstein-Barr virus (EBV) deoxyribonucleic acid (DNA) rose from 4919 to 119,125 copies/ml (Fig. 1a). Pembrolizumab, an anti-PD-1 inhibitor was considered owing to very limited further treatment options. Her archived neck lymph node specimens sent for PD-L1 expression with immunohistochemical staining revealed that the tumor proportion score was 100 and the combined positive score was 101, indicating that a promising response to ICI was expected. She denied any past medical history of tuberculosis. She then received pembrolizumab at $2 \mathrm{mg} / \mathrm{kg}$ every three weeks since September 2016. Her dyspnea and lung metastases improved and reduced in number dramatically after only two cycles of pembrolizumab, accompanied by a slump of EBV DNA to 83 copies/ml (Fig. 1b). She experienced mild irAEs with hypocortisolism and hypothyroidism which were effectively managed with hormone replacement therapy. In May 2018, she presented with a sudden onset of severe, colicky and localized right lower abdominal pain, projectile vomiting and bloody diarrhea with mucus, and persistent fever (temperature $>38.6$ degrees Celsius). Initially immune-related enteritis/colitis was suspected and pembrolizumab was suspended. Positron emission

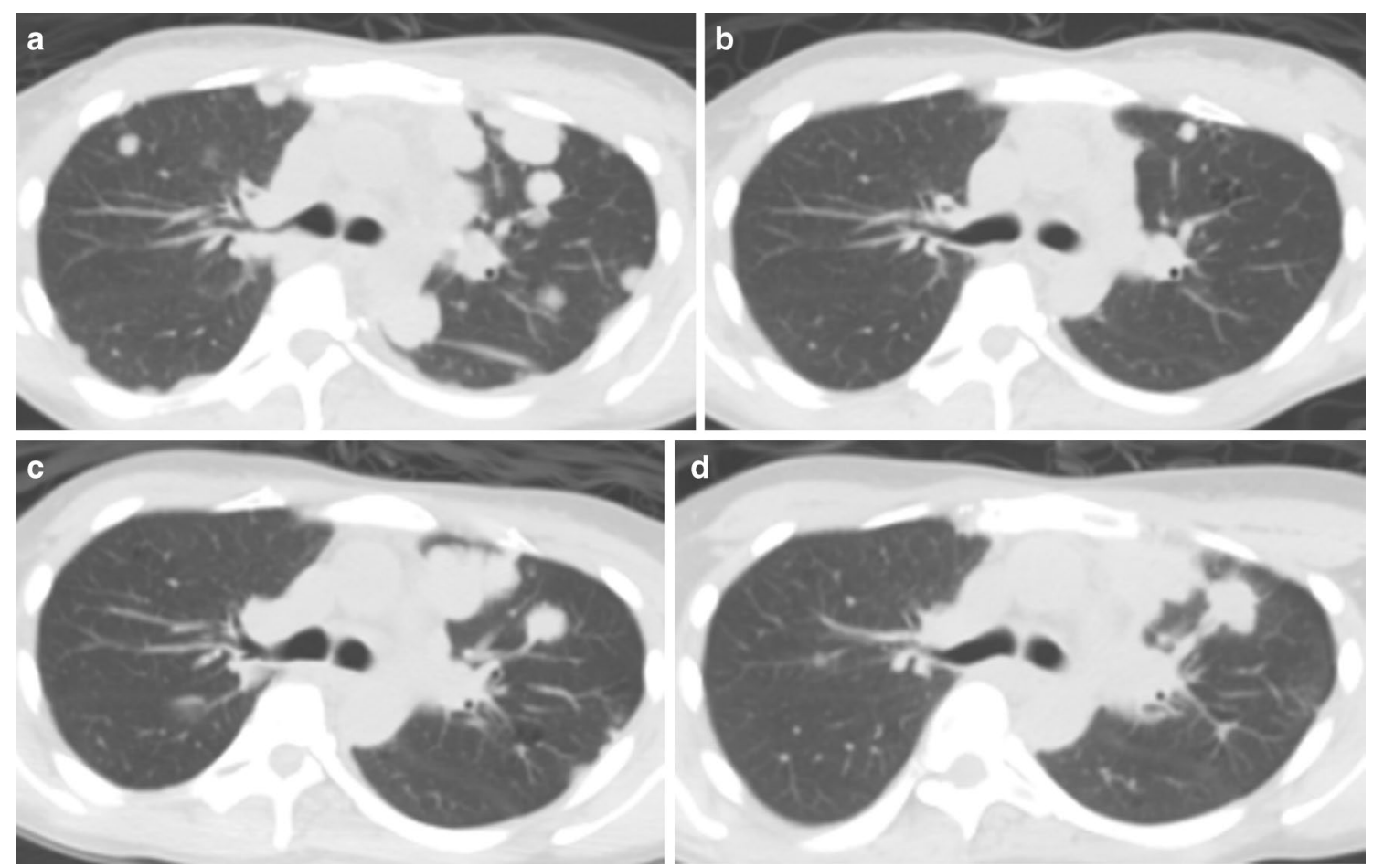

Fig. 1 Serial positron emission tomography with integrated computed tomography images showing response to lung metastases before and after pembrolizumzb treatment, interruption and rechallenge during and after anti-tuberculosis (TB) therapy. a Multiple bilateral lung metastases before pembrolizumab treatment. b Marked reduction in size and number of lung metastases after pembrolizumab treatment. $\mathbf{c}$ Enlarging lung metastases after pembrolizumab interruption due to TB reactivation. $\mathbf{d}$ Tumour shrinkage after re-challenge with pembrolizumab 
tomography with integrated computed tomography (PET-CT) of the abdomen showed terminal ileitis with multiple enlarged mesenteric lymph nodes Colonoscopy was performed and the inflamed terminal ileum was biopsied, which exhibited multiple caseating granulomas with Langerhan cells, compatible with tuberculous ileitis (microscopic images captured by Nikon model DS-FI3 attached to Nikon Eclipse Ni microscope viewed by Application DS-L4 Viewer Software Ver1.2.0) (Fig. 2a). Otherwise, the pathology did not show any features of inflammatory bowel disease or immune-related enteritis. Though Ziehl-Neelsen stain did not identify any acid-fast bacilli, polymerase chain reaction test for $\mathrm{TB}$ of the ileal biopsy and interferon gamma release assay (IGRA) with QuantiFERON TB Gold Plus were both strongly positive. She immediately received anti-TB medication including rifampicin, ethambutol, pyrazinamide and isoniazid for 1 year following our microbiologist's suggestion. Her terminal ileitis and the enlarged mesenteric lymphadenitis resolved in the follow-up PET-CT scan 9 months later. Colonoscopy performed 15 months after the last one confirmed complete resolution of her ileitis (Fig. 2b).

However, her lung metastases worsened again 7 months after anti-TB treatment (Fig. 1c), concurrent with an elevated plasma EBV DNA of 2826 cop$\mathrm{ies} / \mathrm{ml}$ following pembrolizumab interruption. In light of her current progressive metastases and the prior extraordinary response to pembrolizumab, she was re-challenged with pembrolizumab in December 2018 together with her anti-TB treatment. PET-CT scan 5 months later showed promising tumour shrinkage (Fig. 1d). She is still receiving pembrolizumab with no evidence of TB relapse or other irAE.

\section{Discussion and conclusions}

NPC, which is highly associated with EBV infection, is endemic in southern China including Hong Kong with an incidence of more than 20.0 per 100,000 [17]. Platinumbased chemotherapy is the standard first-line treatment for recurrent/metastatic NPC. Unfortunately, almost all patients relapse despite an initial response to chemotherapy. ICI alone, or in combination with systemic chemotherapy has established itself as an efficacious first- and second-line treatment in recurrent/metastatic head and neck squamous cell carcinoma. However, its role in recurrent/metastatic NPC is less defined. The phase I KEYNOTE-028 trial revealed an objective response of $26.3 \%$ with pembrolizumab [18]. Tumor response was highly correlated with PD-L1 expression in tumor cells.

Meanwhile, TB is also a notifiable and endemic disease in Hong Kong, with the notification rate of 54.0 per 100,000 population in 2019 [19], compared to 2.8 per 100,000 in the United States. It may remain latent for years or even decades. Containment of TB in its latent state is achieved through the surveillance of TB-directed $\mathrm{CD} 4+$ and $\mathrm{CD} 8+\mathrm{T}$ cells [20], which happen to be the targets of ICIs. Indeed, the pathophysiology of TB reactivation following ICI therapy is complex and poorly understood, which involves both innate and acquired immune responses [15]. On one hand, exposure to TB leads to increased PD-1 and PD-L1 expression on natural killer (NK) cells of the innate system, resulting in release of interferon- $\gamma$ (IFN $\gamma$ ) and cell lysis. Subsequent binding of PD-1 to PD-L1 and PD-L2 might inhibit further activation of NK cells, which prevents further tissue damage brought by inflammation. On the other hand, TB can also use the acquired immune response to avoid the host immune response by preventing IFNY release
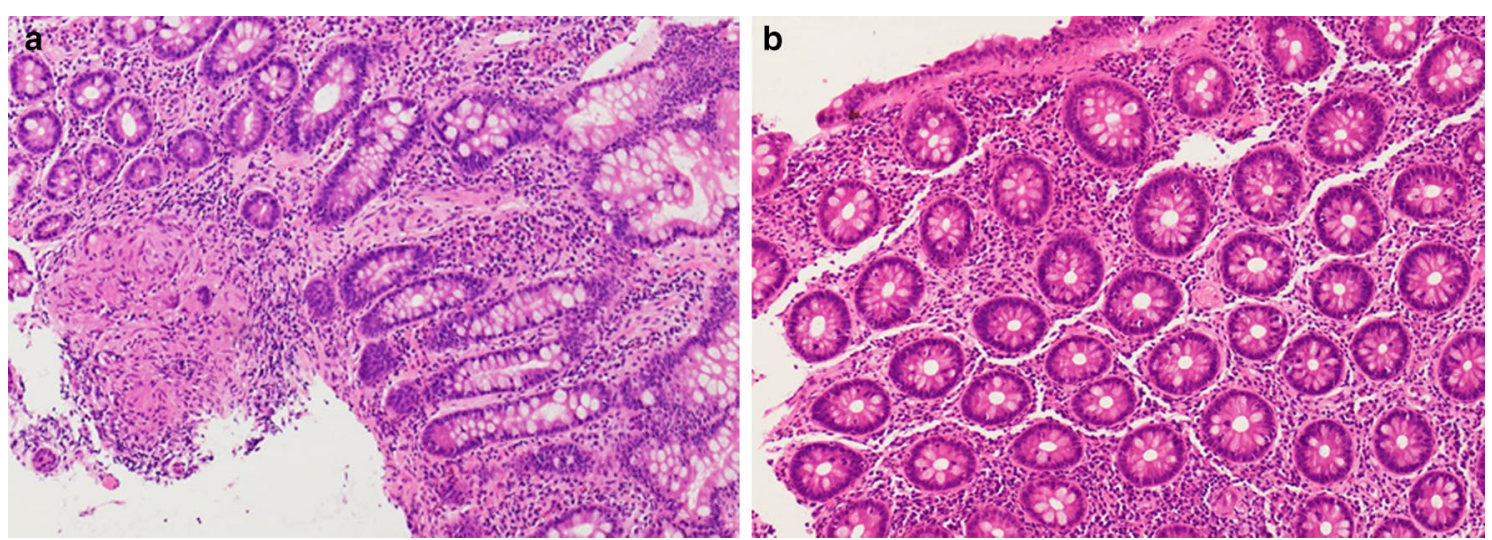

Fig. 2 Ileal biopsies of our patient who developed tuberculosis (TB) reactivation after pembrolizumab and resolution after anti-TB therapy (image resolution $2880 \times 2048$ without additonal processing made). a TB ileitis with small well-formed caseating granulomas after pembrolizumab. b Resolution of TB ileitis after initiation of anti-TB medication and pembrolizumb interruption 
and inhibiting CD8 + cell cytotoxicity via increased PD-1 expression. PD-1/PD-L1 inhibition by ICI, was shown in vitro to enhance $\mathrm{CD} 8+$ cell cytotoxicity against IFN $\gamma$ activated macrophages, leading to $\mathrm{TB}$ reactivation.

There have been some reports of TB reactivation following ICI therapy [3-15]. Intriguingly, patients in these reports who developed $\mathrm{TB}$ reactivation were those whose tumors also responded well to ICIs. It is postulated that $\mathrm{TB}$ reactivation which symbolizes an exaggerated immune response in the host might also predict a favorable tumor response to ICI therapy, though further studies are warranted to confirm such correlation.

A recent retrospective review using the US Food and Drug Administration Adverse Events Reporting System revealed that TB infection was only seen in $0.1 \%$ of patients after PD-1/PD-L1 therapy [16]. That said, the incidence would be much higher in TB-endemic regions. While international and local guidelines on screening TB before initiation of biological agents including anti-tumor necrosis factor- $\alpha$ (TNF $\alpha$ ) for several chronic inflammatory diseases like rheumatoid arthritis have been published [21, 22], consensus on routine screening for TB before ICI therapy for cancer patients is still lacking. Chest radiograph and tuberculin test are recommended screening tools in these guidelines before anti-TNF $\alpha$ therapy. However, they may not be sensitive to diagnose acute/latent TB infection in cancer patients who are often immunocompromised and are also the high-risk groups more susceptible to $\mathrm{TB}$ reactivation after ICI therapy [23].

IGRA by detecting IFN $\gamma$ released by $\mathrm{T}$ cells exposed to TB antigens, has been found more sensitive than the traditional methods to diagnose acute and latent TB, even in immunocompromised patients $[24,25]$. New generations of IGRA were found even more sensitive than their earlier generations [24, 25]. Hopefully, international recommendations on TB screening with accurate and reliable methods before ICI initiation for cancer patients can be proposed and implemented routinely in TB-endemic regions.

In conclusion, we report the first gastrointestinal $\mathrm{TB}$ reactivation following ICI therapy for metastatic NPC. Accurate TB screening methods should be considered for latent TB infection before ICI therapy for cancer patients.

\footnotetext{
Abbreviations

DNA: Deoxyribonucleic acid; EBV: Epstein-Barr virus; ICI: Immune checkpoint inhibitors; IFNy: Interferon- $\gamma$; IGRA: Interferon gamma release assay; irAE: Immune-related adverse events; NK: Natural killer; NPC: Nasopharyngeal carcinoma; PD-1: Programmed death-1; PD-L1: Programmed death-(ligand) 1; PET-CT: Positron emission tomography with integrated computed tomography; TB: Tuberculosis; TNFa: Tumour necrosis factor-a.
}

Acknowledgements

Not applicable.

\section{Authors' contributions}

$\mathrm{KSL}, \mathrm{BMFC}, \mathrm{CFY}$ and VHFL were major contributors in writing this manuscript. KSL, BMCF, KOL, SYC, KM Lam, CFY, IFNH, DLWK, CCT, TWL, MYL, AWML, KKY and VHFL participated in the diagnosis and treatment of the patient, conceptualized the study, and acquired and interpreted the study data. VHFL conceived the study and oversaw the manuscript. All authors read and approved the final manuscript.

\section{Funding}

There was no funding for this study.

\section{Availability of data and materials}

The datasets analyzed during the current study are not publicly available due to patient privacy concerns but are available from the corresponding author on reasonable request.

\section{Declarations}

Ethics approval and consent to participate

Not applicable.

\section{Consent for publication}

The patient provided a written informed consent for the personal and clinical details along with any identifying images to be published in this study.

\section{Competing interests}

The authors declare that they have no competing interests.

\section{Author details}

${ }^{1}$ Department of Clinical Oncology, LKS Faculty of Medicine, The University of Hong Kong, Hong Kong, China. ${ }^{2}$ Department of Pathology, Queen Mary Hospital, Hong Kong, China. ${ }^{3}$ Department of Medicine, LKS Faculty of Medicine, The University of Hong Kong, Hong Kong, China.

Received: 18 January 2021 Accepted: 4 November 2021

Published online: 10 November 2021

\section{References}

1. Sunshine J, Taube JM. PD-1/PD-L1 inhibitors. Curr pin Pharmacol. 2015;23:32-8

2. Michot JM, Bigenwald C, Champiat S, Collins M, Carbonnel F, Postel-Vinay $\mathrm{S}$, et al. Immune-related adverse events with immune checkpoint blockade: a comprehensive review. Eur J Cancer. 2016;54:139-48.

3. Lee JJ, Chan A, Tang T. Tuberculosis reactivation in a patient receiving anti-programmed death-1 (PD-1) inhibitor for relapsed Hodgkin's lymphoma. Acta Oncol. 2016;55(4):519-20.

4. Chu YC, Fang KC, Chen HC, Yeh YC, Tseng CN, Chou TY, et al. Pericardial tamponade caused by a hypersensitivity response to tuberculosis reactivation after anti-PD-1 treatment in a patient with advanced pulmonary adenocarcinoma. J Thorac Oncol. 2017;12(8):e111-4.

5. Fujita K, Uchida N, Horimoto K, Hashimoto M, Nakatani K, Moriyoshi K, et al. Two cases of cavitary lung cancer with concomitant chronic infectious disease. Respir Med Case Rep. 2018;24:122-4.

6. Picchi H, Mateus C, Chouaid C, Besse B, Marabelle A, Michot JM, et al. Infectious complications associated with the use of immune checkpoint inhibitors in oncology: reactivation of tuberculosis after anti PD-1 treatment. Clin Microbiol Infect. 2018;24(3):216-8.

7. Jensen $\mathrm{KH}$, Persson G, Bondgaard AL, Pøhl M. Development of pulmonary tuberculosis following treatment with anti-PD-1 for non-small cell lung cancer. Acta Oncol. 2018;57(8):1127-8.

8. He W, Zhang X, Li W, Kong C, Wang Y, Zhu L, et al. Activated pulmonary tuberculosis in a patient with melanoma during PD-1 inhibition: a case report. OncoTargets Ther. 2018;11:7423-7.

9. Takata S, Koh G, Han Y, Yoshida H, Shiroyama T, Takada H, et al. Paradoxical response in a patient with non-small cell lung cancer who received nivolumab followed by anti-Mycobacterium tuberculosis agents. J Infect Chemother. 2019;25(1):54-8. 
10. Tsai CC, Chen JH, Wang YC, Chang FY. Re-activation of pulmonary tuberculosis during anti-programmed death-1 (PD-1) treatment. QJM. 2019;112(1):41-2.

11. van Eeden R, Rapoport BL, Smit T, Anderson R. Tuberculosis infection in a patient treated with nivolumab for non-small cell lung cancer: case report and literature review. Front Oncol. 2019;9:659.

12. Inthasot V, Bruyneel M, Muylle I, Ninane V. Severe pulmonary infections complicating nivolumab treatment for lung cancer: a report of two cases. Acta Clin Belg. 2020;75(4):308-10.

13. Barber DL, Sakai S, Kudchadkar RR, Fling SP, Day TA, Vergara JA, et al. Tuberculosis following PD-1 blockade for cancer immunotherapy. Sci Transl Med. 2019;11(475):eaat2702.

14. Song JS, Jeffery CC. Laryngeal tuberculosis in a patient on avelumab for metastatic nasopharyngeal carcinoma. J Immunother. 2020;43(7):222-3.

15. Langan EA, Graetz V, Allerheiligen J, Zillikens D, Rupp J, Terheyden P. Immune checkpoint inhibitors and tuberculosis: an old disease in a new context. Lancet Oncol. 2020;21(1):e55-65.

16. Anand K, Sahu G, Burns E, Ensor A, Ensor J, Pingali SR, et al. Mycobacterial infections due to PD-1 and PD-L1 checkpoint inhibitors. ESMO Open. 2020:5(4):e000866.

17. Bray F, Ferlay J, Soerjomataram I, Siegel RL, Torre LA, Jemal A. Global cancer statistics 2018: GLOBOCAN estimates of incidence and mortality worldwide for 36 cancers in 185 countries. CA Cancer J Clin. 2018;68(6):394-424

18. Hsu C, Lee SH, Ejadi S, Even C, Cohen RB, Le Tourneau C, et al. Safety and antitumor activity of pembrolizumab in patients with programmed death-ligand 1-positive nasopharyngeal carcinoma: results of the KEYNOTE-028 study. J Clin Oncol. 2017;35(36):4050-6.
19. Centre for Health Protection. Notification \& death rate of tuberculosis (all forms), 1947-2019. Available from: https://www.chp.gov.hk/en/statistics/ data/10/26/43/88.html. Assessed 30 Dec 2020.

20. O'Garra A, Redford PS, McNab FW, Bloom Cl, Wilkinson RJ, Berry MP. The immune response in tuberculosis. Annu Rev Immunol. 2013;31:475-527.

21. British Thoracic Society Standards of Care Committee. BTS recommendations for assessing risk and for managing Mycobacterium tuberculosis infection and disease in patients due to start anti-TNF-alpha treatment. Thorax. 2005;60(10):800-5.

22. Wang PH, Lin $\mathrm{CH}$, Chang TH, Wu CS. Chest roentgenography is complementary to interferon-gamma release assay in latent tuberculosis infection screening of rheumatic patients. BMC Pulm Med. 2020;20(1):232.

23. Kim EY, Lim JE, Jung JY, Son JY, Lee KJ, Yoon YW, et al. Performance of the tuberculin skin test and interferon-gamma release assay for detection of tuberculosis infection in immunocompromised patients in a BCG-vaccinated population. BMC Infect Dis. 2009;9:207.

24. Della Bella C, Spinicci M, Alnwaisri HFM, Bartalesi F, Tapinassi S, Mencarini J, et al. LIOFeronTB/LTBI: A novel and reliable test for LTBI and tuberculosis. Int J Infect Dis. 2020;91:177-81.

25. Sotgiu G, Saderi L, Petruccioli E, Aliberti S, Piana A, Petrone L, et al. QuantiFERON TB Gold Plus for the diagnosis of tuberculosis: a systematic review and meta-analysis. J Infect. 2019;79(5):444-53.

\section{Publisher's Note}

Springer Nature remains neutral with regard to jurisdictional claims in published maps and institutional affiliations.
Ready to submit your research? Choose BMC and benefit from:

- fast, convenient online submission

- thorough peer review by experienced researchers in your field

- rapid publication on acceptance

- support for research data, including large and complex data types

- gold Open Access which fosters wider collaboration and increased citations

- maximum visibility for your research: over $100 \mathrm{M}$ website views per year

At BMC, research is always in progress.

Learn more biomedcentral.com/submissions 